\title{
Giant unilamellar vesicles (GUVs) as a new tool for analysis of caspase-8/Bid-FL complex binding to cardiolipin and its functional activity
}

\author{
O Jalmar ${ }^{1}$, AJ García-Sáez ${ }^{2}$, L Berland ${ }^{3}$, F Gonzalvez ${ }^{4}$ and PX Petit ${ }^{\star, 1}$
}

Cardiolipin (CL) has recently been shown to be both an anchor and an essential activating platform for caspase-8 on mitochondria. These platforms may be at the mitochondrial contact sites in which truncated Bid (tBid) has been demonstrated to be located. A possible role for CL is to anchor caspase-8 at contact sites (between inner and outer membranes), facilitating its self-activation, Bid-full length (FL) cleavage, tBid generation (and Bax/Bak activation and oligomerization), mitochondrial destabilization and apoptosis. We have developed an in vitro system that mimics the mitochondrial membrane contact site platform. This system involves reconstituting caspase-8, Bid-FL and CL complexes in giant unilamellar vesicles (GUVs). We first validated the system by flow cytometry analysis of light-scattering properties and nonyl acridine orange staining of their CL content. Then, we used flow cytometry analysis to detect the binding of active caspase-8 to CL and the subsequent truncation of bound Bid-FL. The tBid generated interacts with $\mathrm{CL}$ and induces GUV breakage and partial re-vesiculation at a smaller size. Our findings suggest an active role for mitochondrial membrane lipids, particularly $C L$, in binding active caspase-8 and providing a docking site for Bid-FL. This phenomenon was previously only poorly documented and substantially underestimated.

Cell Death and Disease (2010) 1, e103; doi:10.1038/cddis.2010.81; published online 2 December 2010

Subject Category: Experimental Medicine

Fas (also called APO-1 or CD95) and its physiological ligand, FasL, regulate apoptosis of unwanted or dangerous cells, thereby functioning as a defense against autoimmunity and cancer development. ${ }^{1-4}$ The mechanism by which the 'death receptor' FAS triggers the apoptosis of cells depends on the cell type. ${ }^{1-4}$

In type I cells, Fas-induced activation of caspase-8 leads to activation of effector caspases and subsequently cell death. In type II cells, including Barth syndrome-derived hepatocytes and pancreatic $\beta$-cells, cell death requires caspase cascade amplification through caspase-8-mediated activation of the proapoptotic Bcl-2 family member Bid-FL (BH3 interacting domain death agonist-full length) ${ }^{5-7}$ Caspase-8 cleaves Bid$\mathrm{FL}$, which is a member of the $\mathrm{Bcl}-2$ family, and the resulting truncated Bid (tBid) activates the mitochondria. ${ }^{8,9}$ Mitochondria are activated in both type I and type II cells, but this activation is not strictly necessary for the death of type I cells. Upon activation, mitochondria release pro-apoptotic molecules, including cytochrome $c$ and Smac/DIABLO. ${ }^{10}$ Once in the cytosol, cytochrome $c$ induces the activation of the apoptosome leading to the activation of further downstream caspases and the end result is apoptosis.
The importance of specific protein-lipid interactions for the proper functioning of an organelle is clearly exemplified within the inner membrane (IMM) of mitochondria, in which most of the unique phospholipid, cardiolipin (CL), is found.$^{11} \mathrm{CL}$ is associated with all of the major players in oxidative phosphorylation, including complexes I, III, IV and V, and the major carrier proteins for adenine nucleotides and phosphates. ${ }^{12}$ The absence of $C L$ results in structural abnormalities in the organization of complexes that have direct functional consequences. CL has been described to be enriched by 10 to $20 \%$ in the contact site between the two mitochondrial membranes. ${ }^{13} \mathrm{CL}$ is a structurally unusual phospholipid with one negative charge at physiological $\mathrm{pH}$ associated with its two headgroups and four associated fatty acyl chains. ${ }^{14} \mathrm{CL}$ is synthesized by $C L$ synthase, in the matrix-facing leaflets of the IMM. ${ }^{9}$ Newly synthesized $C L$ undergoes a remodeling process during which saturated acyl chains are replaced with more unsaturated chains, thereby establishing a high degree of acyl chain symmetry.

$\mathrm{CL}$ is associated with several mitochondrial-dependent apoptotic steps, ${ }^{15}$ including the proapoptotic function of Bcl-2

\footnotetext{
${ }^{1}$ Institut Cochin, CNRS UMR 8104, INSERM U1016, Université Paris V - R. Descartes, 24 rue du Faubourg Saint-Jacques, Paris 75014, France; ${ }^{2}$ German Cancer Research Center and Max-Planck Institute for Metals Research, Bioquant, Im Neuenheimer Feld 267, Heidelberg 69120, Germany; ${ }^{3}$ Institut Curie, Centre de Recherche, Laboratoire Physico-Chimie (UMR CNRS 168) 11 rue Pierre et Marie Curie, Paris Cedex 0575231 , France and ${ }^{4}$ Genentech, Postdoctoral Research Fellow, 1 DNA Way, MS 42, South San Francisco, CA 94080, USA

${ }^{*}$ Corresponding author: PX Petit, Institut Cochin, CNRS UMR 8104, INSERM U 1016, Université Paris V - R. Descartes, 24 Rue du Faubourg Saint-Jacques, Paris 75014, France. Tel: + 3314441 2450; Fax: + 3314441 2421; E-mail: patrice.petit@inserm.fr

Keywords: apoptosis; Bcl-2 family members; Bid-FL; caspase-8; cardiolipin; giant unilamellar vesicles

Abbreviations: Bid-FL, BH3-interacting domain death agonist-full length; CL, cardiolipin; DED, death effector domain; DISC, death-inducing signaling-complex; GUVs, giant unilamellar vesicles; IMM, inner membrane; NAO, nonyl acridine orange; OMM, outer membrane; p55, full-length pro-caspase-8; p43/p10, first cleaved form of caspase-8; p18/p10, fully cleaved form of caspase-8; PC, phosphatidyl choline; tBid, active C-terminal region of Bid or truncated Bid

Received 10.6.10; revised 23.9.10; accepted 06.10.10; Edited by V De Laurenzi
} 
family proteins. CL can serve as a docking site for $\mathrm{tBid}^{16}$ and is subsequently required for Bax activation and the destabilization of mitochondrial membrane structure and function. ${ }^{17}$ By anchoring cytochrome $c$ to the IMM, CL both facilitates the electron transport chain and hinders cytochrome $c$ release during apoptosis, evidence that complete release of cytochrome $c$ requires the disruption of its interactions with CL. CL peroxidation and degradation facilitates such disruption. ${ }^{18}$

Pro-caspase- 8 is mostly in the cytosol, but is also loosely associated with the mitochondrial outer membrane (OMM). ${ }^{19,20}$ We recently reported that Fas receptor activation induced the translocation of active caspase- 8 to the mitochondrial surface, a step that was dependent on the presence of mature $\mathrm{CL}$ in the OMM. ${ }^{21}$ This translocation of caspase-8 was necessary for the activation and amplification of caspase-8 processing. Immortalized lymphoblasts from a Barth syndrome patient containing non-mature CL exhibit a blockage of the signal transduction through mitochondria, showing that $C L$ is required for this process.

Our discovery of an activation platform formed by CL/ Caspase-8/Bid-FL at mitochondrial contact sites ${ }^{21}$ opens various possibilities for research. In particular, more detailed biophysical analysis of the behavior of active caspase- 8 and of Bid-FL binding is needed to understand the interactions between the various components of the platform.

Here, we describe the development of an experimental biomimetic system as a model for studying interactions between proteins (active caspase- 8 and Bid-FL) and the mitochondrial OMM. It also allows the development of tools for measuring the effects of binding between the components.

Our approach was to identify a minimal physico-chemical system that models mitochondrial contact sites and the interaction of caspase-8/Bid-FL with CL. It involves giant unilamellar vesicles (GUVs) as model membrane system. We first validated flow cytometry for the analysis of GUVs on a routine basis. Then, we show how flow cytometry measurements can be used to measure binding between GUVs and putative components constituting the functional enzymatic platform.

\section{Results}

GUV analysis by flow cytometry. Physiological membrane systems, such as mitochondrial membranes and their contact sites, are extremely complex. To develop an experimental model of such systems, we used a cell-free reconstituted system constituted of GUVs with defined phospholipid composition. These are the membrane systems that have the advantages of being freestanding bilayers a few to hundred micrometers in diameter, suitable for flow cytometry analysis (Figure 1) and confocal microscopy.

We used this experimental model to study the interactions between $\mathrm{CL}$ and other highly purified proteins. First, we validated the use of flow cytometry for the study of GUVs. The light-scattering properties of GUVs were used to follow vesicle size changes, and NAO staining was used to measure their CL content (Figure 1). Beads of defined size were added to GUV samples to facilitate measuring GUV size (Figure 1a). Most of the GUVs were larger than the $10 \mu \mathrm{m}$ beads, and in the range 10 to $100 \mu \mathrm{m}$ in diameter.
The GUVs were homogeneously stained by NAO with the labeling intensity depending on their size (Figure 1b) and $\mathrm{CL}$ content (Figure 1c). There was a linear relationship between CL content and NAO fluorescence. We examined GUVs of various compositions by confocal imaging. This confirmed the staining of GUVs containing CL by NAO and weak fluorescence of GUVs devoid of CL (Figure $1 \mathrm{c}-\mathrm{e}$ ). The vesicles with $90: 10 \mathrm{PC} / \mathrm{CL}$ composition appeared round and mostly devoid of internal structures. They were also easy to handle. We, therefore, used this GUVs composition for all subsequent experiments, except where indicated otherwise.

tBid binds to GUVs containing CL. tBid induces the destabilization of mitochondrial membranes and changes in their functional activity ${ }^{17,22}$ by binding to the CL at contact sites. This binding depends essentially on the interaction between the hairpin-forming domain of tBid and CL both. ${ }^{24}$ This also appears to be the case when using CL-containing phospholipid monolayers. $^{25}$

We investigated the binding and effects of tBid on GUVs containing or not containing CL. tBid did not bind to vesicles containing only phosphatidylcholine (Figure $2 a, b$, e and $f$ ), but showed specific binding to GUVs containing CL ((Figure 2c, d, $\mathrm{g}$ and $\mathrm{h}$ ). This is consistent with previous findings concerning CL-mediated selectivity of tBid interactions with lipid membranes. ${ }^{16,22,25-28}$ The weak non-specific binding did not modify the light scatter or the fluorescence of PC-only-GUVs (Figure $2 b$ and $f$ ). We then investigated the consequences of this tBid binding for the light-scattering properties of CL-GUVs (Figure 2c and $\mathrm{g}$ ). The addition of $20 \mathrm{nM}$ tBid to CL-GUVs (online addition) was immediately followed by tBid binding and a substantial change in light-scattering properties. The GUVs became smaller and the SSC increased. Indeed, the CL-GUVs broke down and re-vesiculated at smaller sizes (39\% under $10 \mu \mathrm{m}$ ) than the initial and control CL-GUVs vesicles (29\% under $10 \mu \mathrm{m})$. The tBid fluorescence was clearly associated with the low SCC vesicle population (Figure $2 \mathrm{~h}$ ).

Active caspase-8 preferentially binds CL-containing GUVs. The activation of caspases often involves the formation of triggering complexes. Many such complexes, or activation platforms, have been described in the vicinity of the plasma membrane: the death-inducing signaling-complex (DISC) for caspase-8 and $-10,{ }^{29}$ the apoptososme for caspase $-9,{ }^{30}$ the PIDDosome for caspase- 2 and the inflammasome for caspase- 1 and $-5 .^{31}$ The auto-processing of caspase- 8 into fully active caspase- 8 at the DISC involves two steps. First, upon homodimerization, pro-caspase-8 (p55) is cleaved into two active subunits ( $p 43$ and p10). The second cleavage is in p43 between the DED and the p18 subunits to generate the fully active p18/p10 form. The first cleavage product, $\mathrm{p} 43 / \mathrm{p} 10$, associates with $\mathrm{CL}$-containing membranes and is resistant to alkaline wash, suggesting that it is inserted into the mitochondrial OMM. ${ }^{21}$ Indeed, this was the first proof of caspase- $8^{32}$ integration into the mitochondrial membrane. ${ }^{16}$ The resistance to alkaline washing suggests that hydrophobic interactions with the unsaturated acyl chain of CL may contribute to the stability of the complex 

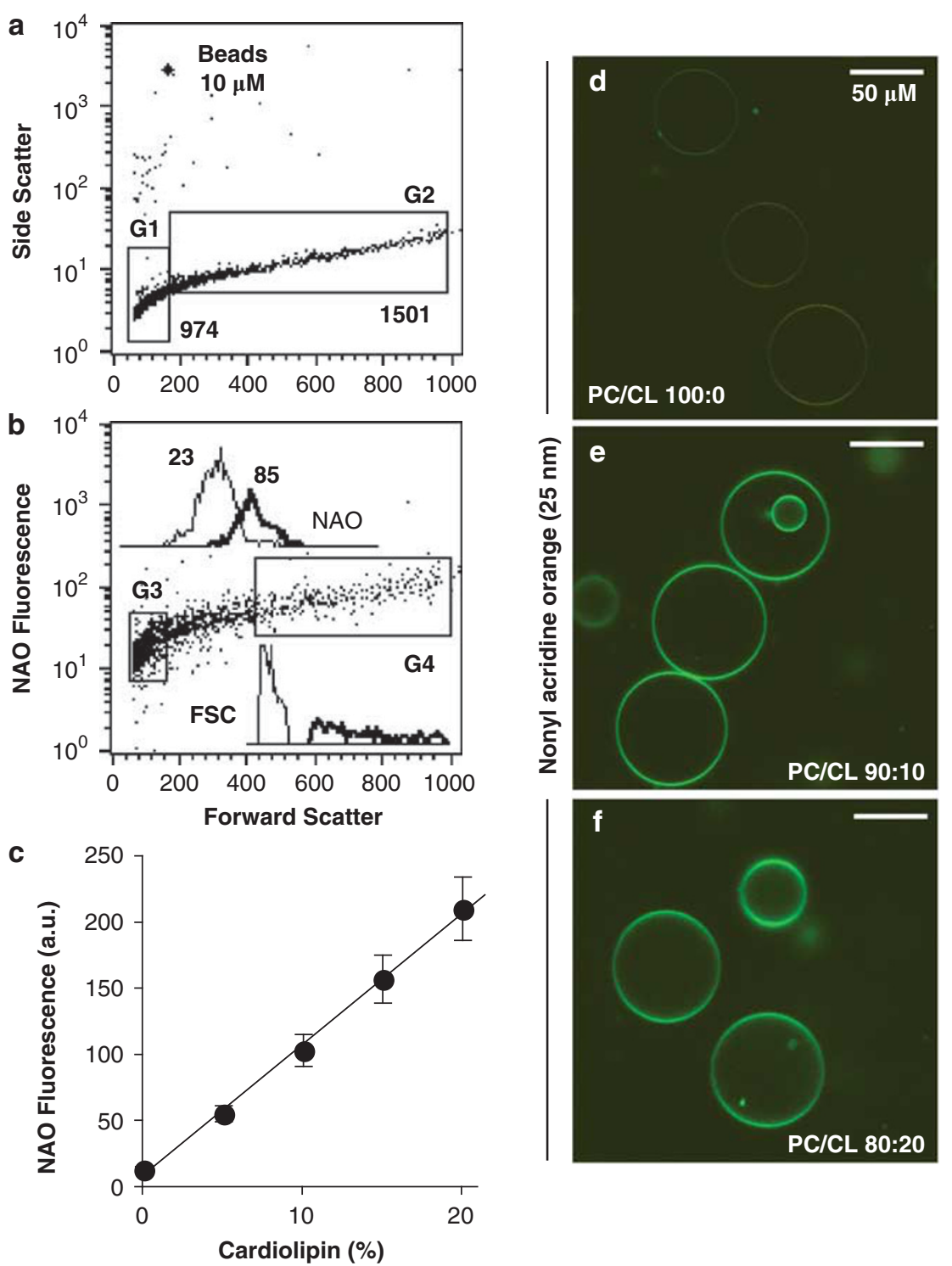

Figure 1 Flow cytometry analysis of GUVs and nonyl acridine orange staining of CL. (a) Light-scattering properties (FSC/SSC) of a GUV preparation composed of 90 : 10 PC/CL. Fluorescent beads $(10 \mu \mathrm{m})$ were included in the sample as size standards. Gates G1 and G2 define two GUV subpopulations, with G1 for GUVs with FSC lower than beads and G2 for GUVs with FSC higher than the beads (the GUVs in this sample are between 10 to $90 \mu \mathrm{M}$ across). The number of GUV in each subpopulation is given. (b) Biparametric histograms of FSC versus NAO fluorescence. The histograms of fluorescence (top left) and FSC (bottom right) are given for the G3 and G4 populations. (c) Relationship between GUV cardiolipin content and NAO fluorescence. All data reported correspond to analysis of 2500 events. The values for mean fluorescence are given in arbitrary units (a.u.) with S.D. $(n=10)$. (d-f) Confocal microscopy of GUVs with different CL contents. (d) PC only, (e) PC/CL $90: 10$ and (f) PC/CL $80: 20$

formed by CL and p18/p10. However, such bound caspase-8 is sensitive to trypsin proteolysis indicating that it remains accessible from the cytosol. ${ }^{21}$

We investigated the selectivity of the interaction between caspase-8 and GUVs of different lipid compositions (Figure 3). Vesicles containing and those not containing CL exhibited similar autofluorescence (Figure $3 a$ and $c$ ). As added caspase-8 is not fluorescent, we used an indirect measurement with FITC-VAD-fmk as a reporter of the presence of the active caspase- 8 at the GUV surface (Figure 3 and Table 1). The binding of active caspase- 8 to CL-GUVs was almost seven times greater than that to PC-only-GUVs (fluorescence of 853 and 136, respectively, Figure $3 b$ and d). The signal for PC-only-GUVs presumably relflect non-specific binding. The fixation of the fluorescent reporter molecule (FITC-VAD$\mathrm{fmk}$ ) to the active site of caspase-8 molecules can be inhibited by z-VAD-fmk, a widely used pan-caspase inhibitor. z-VADfmk did not affect autofluorescence or non-specific binding. We tested the effects of this and other caspase inhibitors on FITC-VAD-fmk staining of bound active caspase-8 (Table 1). Both the general caspase inhibitors (z-VAD-fmk and Boc-D$\mathrm{fmk}$ ) and the specific caspase-8 inhibitor (z-IETD-fmk) effectively abolished FITC-VAD-fmk staining of caspase-8 linked to GUVs. Bcl- $\mathrm{X}_{\mathrm{L}} \Delta \mathrm{CT},{ }^{33}$ which binds vesicles independently of the $C L$ content, did not interfere with this staining (Table 1). 


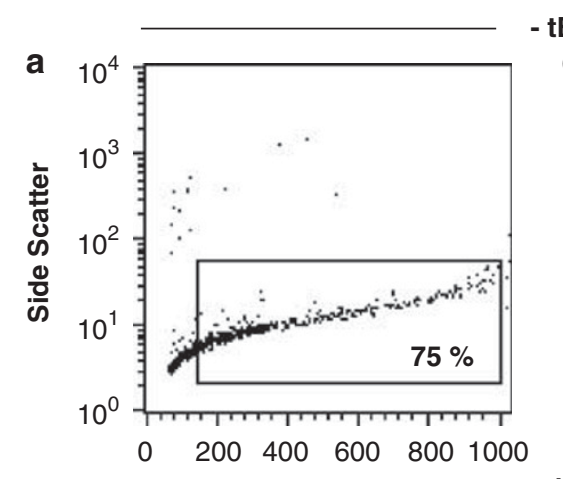

- tBid

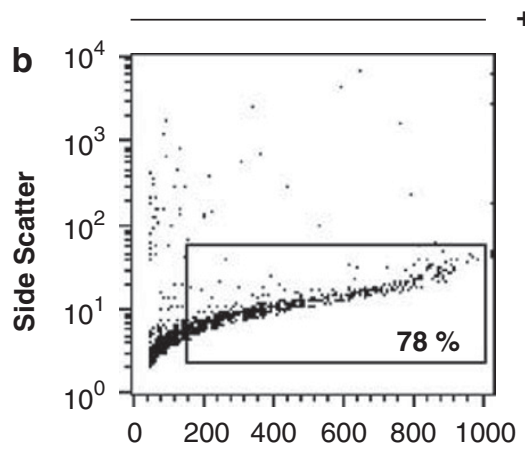

e $10^{4}$

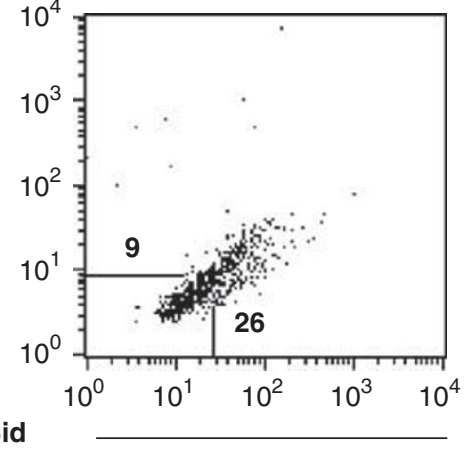

+ tBid

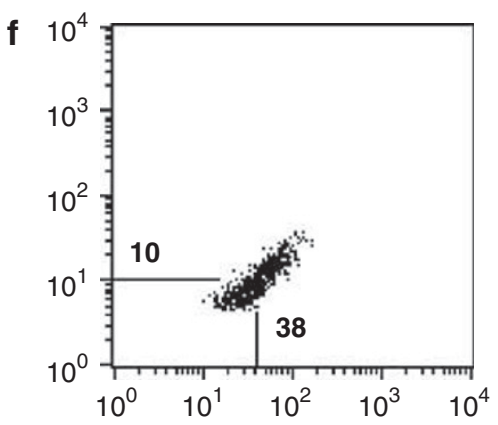

윰

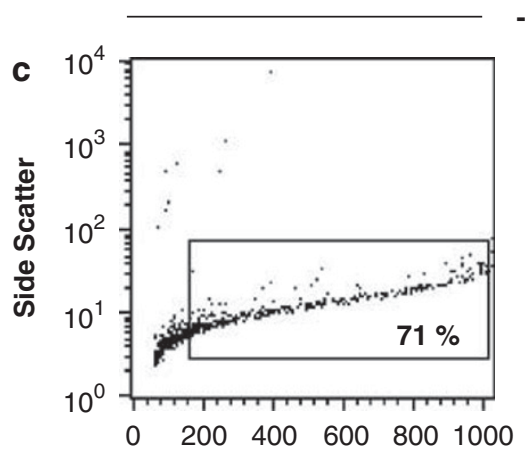

- tBid
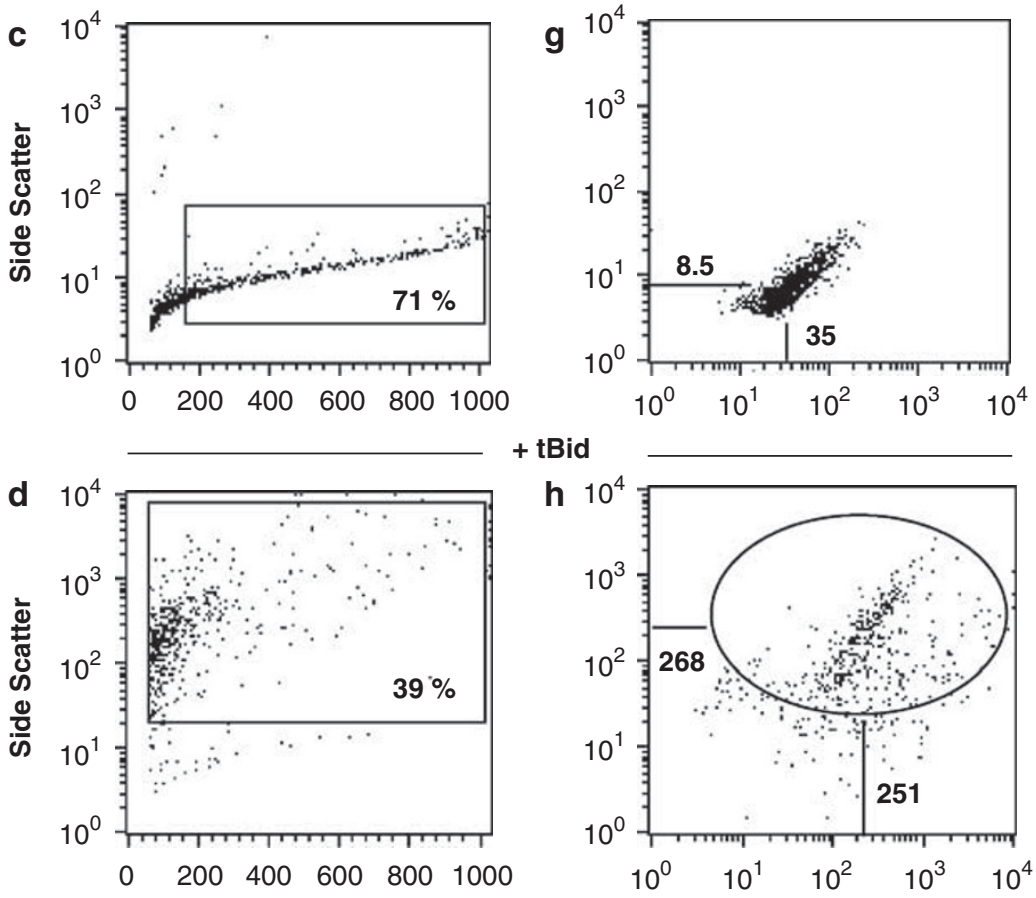

Forward Scatter

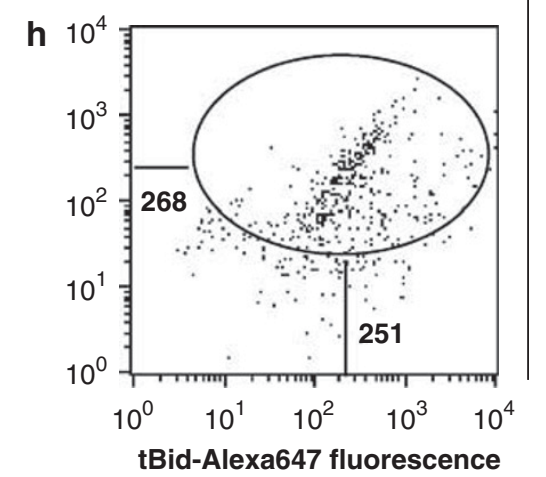

음

Figure 2 Effets of tBid binding to GUVs containing CL. GUVs constituted of PC only (panels $\mathbf{a}, \mathbf{b}, \mathbf{e}$ and $\mathbf{f}$ ) or of PC/CL $90: 10$ (c, $\mathbf{d}, \mathbf{g}$ and $\mathbf{h}$ ) were studied. The GUVs were treated or not treated with $10 \mathrm{nM}$ tBid. (a-d) Light-scattering properties of the GUVs (FSC/SCC). The percentage of vesicles in each gated population is shown. (e-h) tBidBodipy Alexa647 versus SSC. The values reported are the mean SSC versus the mean Alexa fluorescence of the selected populations (a-d)

Formation of a functional enzymatic complex between caspase-8, Bid-FL and CL. One hypothesis is that, CL provides an activation platform upon which caspase-8 can cluster, oligomerize and be processed into both p43/p10 and p18/p10. Indeed, the amount of caspase-8 accumulating at the DISC in type II cells is very small and even not detectable by western blotting; this led to the suggestion that a mitochondrial step is necessary for amplification (i.e., caspase- 8 accumulation at the mitochondrial membrane level and recruitment of Bid-FL). ${ }^{34}$ The $\mathrm{p} 43 / \mathrm{p} 10$ on the 

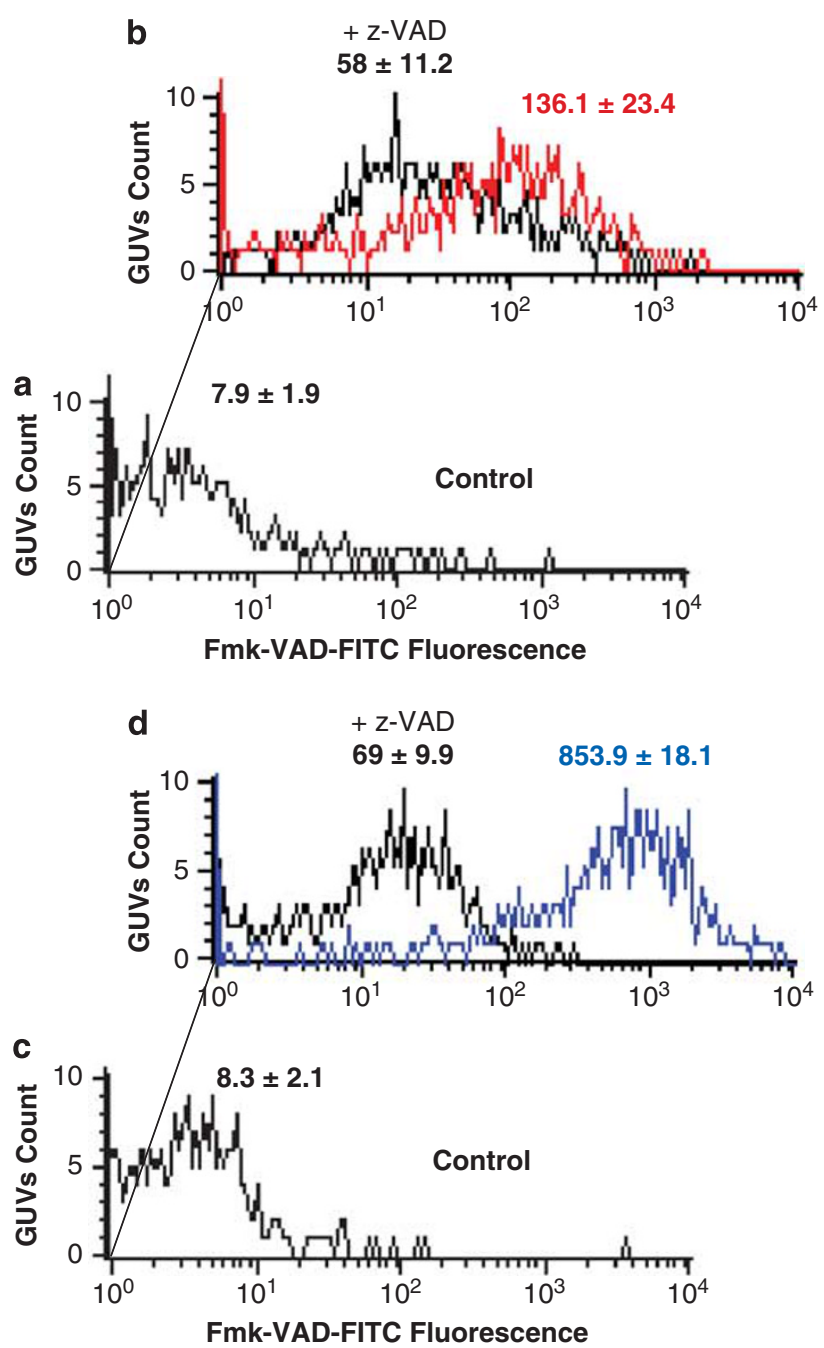

Figure 3 Flow cytometry analysis of caspase-8 binding to GUVs containing CL. FITC-VAD-fmk ( $500 \mathrm{nM})$, a fluorescent marker of the caspase-8 active site, was used to stain caspase-8 bound to the vesicles. (a) PC-only-GUV control (autofluorescence at $488 \mathrm{~nm}$ excitation). (b) Binding of caspase-8 to PC-only-GUVs: red line, in the absence of Z-VAD-fmk (non-fluorescent); black line, following preincubation with $5 \mu \mathrm{M} z-V A D-f m k$. (c) CL-GUV (composition 90:10 PC/CL) control (autofluorescence). (d) Binding of caspase-8 to GUVs containing CL (90:10 PC/ $\mathrm{CL}$ ): blue line, in the absence of z-VAD-fmk; black line, after preincubation with $5 \mu \mathrm{M}$ $z$-VAD-fmk. Reported values are mean fluorescence and S.D.s for 2500 events involving GUVs over $10 \mu \mathrm{m}$ in diameter $(n=11)$

outer mitochondrial membrane is cross-linked to other $\mathrm{p} 43 /$ p10 molecules to form high-molecular weight oligomers, reinforcing this view. ${ }^{21}$ This also suggests that caspase-8 processing to its active form ( $18 / \mathrm{p} 10$ ) occurs on the mitochondria and involves a CL-dependent system. Bid-FL (the substrate), which does not bind to $\mathrm{CL}$, may then be brought together with caspase-8 (the active enzyme) at this site in which the interaction produces tBid (the product).

To test this hypothesis, we first targeted active caspase- 8 to PC-only- and CL-GUVs and then added Alexa-labeled Bid-FL (Figure 4). The system was highly non-saturable in the presence of $\mathrm{CL}$ and caspase-8. Caspase- 8 addition did not modify either the light scatter or the autofluorescence of the GUVs (Figure 4a and e). The immediate measurements
Table 1 Staining of caspase-8 bound to GUVs with CaspACE in the presence or absence of caspase inhibitors

\begin{tabular}{llc}
\hline CaspACE & $\begin{array}{c}\text { Caspase-8 } \\
\text { inhibitors }\end{array}$ & $\begin{array}{c}\text { Mean fluorescence } \\
\text { in A.U. }\end{array}$ \\
\hline FITC-VAD-fmk & - & $7.5 \pm 1.9$ \\
FITC-VAD-fmk & IETD-fmk & $138.9 \pm 24.5$ \\
FITC-VAD-fmk & Z-VAD-fmk & $35.9 \pm 6.9$ \\
FITC-VAD-fmk & Boc-D-fmk & $43.5 \pm 7.9$ \\
& $\begin{array}{l}\text { Anti-apoptotic } \\
\text { family member }\end{array}$ \\
FITC-VAD-fmk & Bcl- $\mathrm{X}_{\mathrm{L}} \Delta \mathrm{Ct}$ & \\
\hline
\end{tabular}

The GUVs were preincubated for $15 \mathrm{~min}$ with or without caspase inhibitors and then analyzed by flow cytometry. The fluorescent marker FITC-VAD-fmk $(500 \mathrm{nM})$ was added on-line and measurements were made immediately. All caspase inhibitors and the anti-apoptotic family member $\mathrm{Bcl}-\mathrm{X}_{\mathrm{L}} \Delta \mathrm{Ct}$ $(250 \mathrm{~nm})$ were pre-incubated with the samples for $30 \mathrm{~min}$ before addition of FITC-VAD-fmk.

$(t=0)$ correspond to the fluorescence of Bid-FL on CL-GUVs (Figure $4 \mathrm{~b}$ and $\mathrm{f}$ ). In less than two minutes, two populations were distinguishable in terms of light scattering and of Bid-FLAlexa647 (nm) fluorescence (Figure $4 \mathrm{c}$ and g). The fluorescence of vesicles of high FSC and low SSC (25-30\% of the GUVs) was similar to that of the stained control population, whereas a new population of low FSC and high SSC (51\%) appeared with a higher fluorescence (Figure 4g). Possibly, the enzymatic system was rapidly active in the presence of the three components (CL/Caspase-8 and Bid-FL), all together in the vicinity of the vesicles, such that tBid was generated and active (similar to the effect of tBid described in Figure 2d and h). After $45 \mathrm{~min}$, only small GUVs with high SCC were detected in significant abundance $(30 \%$ of all objects detected). They exhibit weak fluorescence evidence of the cleavage of the fluorescent Bid-FL-Alexa647 (nm) (Figure 4i) to generate tBid (non-fluorescent). In parallel, there was re-vesiculation of the GUVs at smaller size (low FSC) and with larger side scatter (high SSC) (Figure 4d) and complete destruction of some (not directly visualized on histograms because the threshold imposed on the FSC, but nevertheless calculable). The sequence of events is schematized in Figure 5, together with a statistical analysis of the generation of small vesicles (low FSC/high SSC) from the initial population of GUVs (high FSC/low SSC). It is clear that the amount of small vesicles generated is smaller than the number of GUVs initially present ( $75 \pm 7 \%$ of the total events). Figure $5 b-d$ shows the various subpopulations as revealed by confocal analysis of CL-GUVs stained red with a lipid marker (and incubated with caspase-8 and Bid-FL-Bopipy $488 \mathrm{~nm}$ ); the types of vesicles visualized included normal-sized roundshaped vesicles (over $10 \mu \mathrm{m} \mathrm{FSC} \mathrm{high/SSC} \mathrm{low)} \mathrm{(Figure} \mathrm{5b),}$ small round vesicles (below $10 \mu \mathrm{m}$ FSC low/SSC high) (Figure 5c) and various fluorescent debris that had a tendency to aggregate (Figure $5 \mathrm{~d}$ ).

\section{Discussion}

Given that the amount of active caspase-8 generated at the DISC during type II cell apoptosis is very low, it seems likely that the mitochondrial steps are necessary for the amplification of the apoptotic signals required for extrinsic 

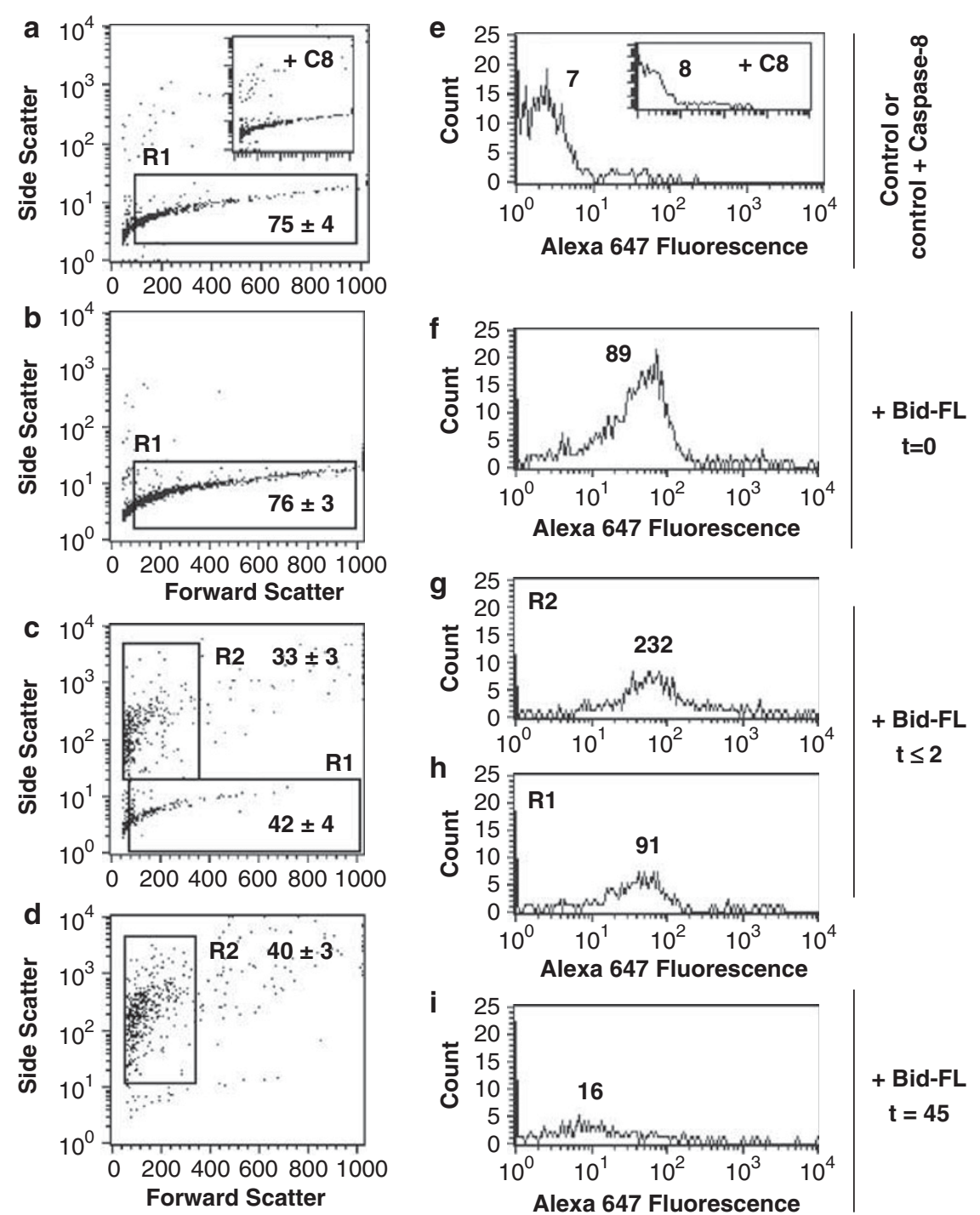

+ Bid-FL

$\mathbf{t} \leq 2$
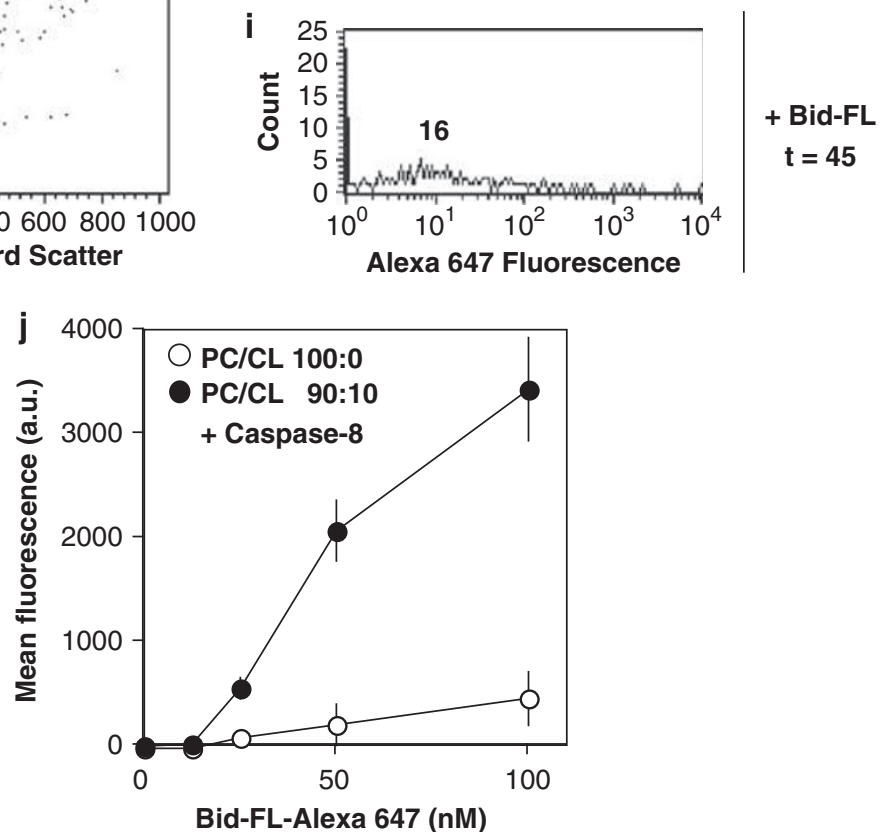

Figure 4 Reconstitution of the caspase-8/Bid/CL platform in GUVs. (a-i) Kinetics of Bid-FL activity on CL-GUVs in presence of active caspase-8. CL-containing GUVs $(90: 10 \mathrm{PC} / \mathrm{CL}$ ) pre-treated with $100 \mathrm{nM}$ of active caspase-8 were incubated with Alexa647-labeled Bid-FL. Light-scattering properties (FSC/SSC, panels a-d) and Alexa647 fluorescence (panels e-i) of the GUVs were measured by flow cytometry at time 0,2 and $45 \mathrm{~min}$ after incubation. Inserts in panel $\mathbf{a}$ and $\mathbf{e}$ represent the effect of the addition of active caspase-8 on GUVs. The data are the mean of 12 experiments. (j) CL promotes the binding of Bid-FL to the GUVs in presence of caspase-8 (100 nM). PC/CL (100:0) and PC/CL (90:10) containing GUVs were pretreated with $100 \mathrm{nM}$ of active caspase-8 and incubated with increasing concentration of Alexa647-labeled Bid-FL (12.5, 25, 50 and $100 \mathrm{nM}$ ). Alexa647 fluorescence of the GUVs was measured by flow cytometry 


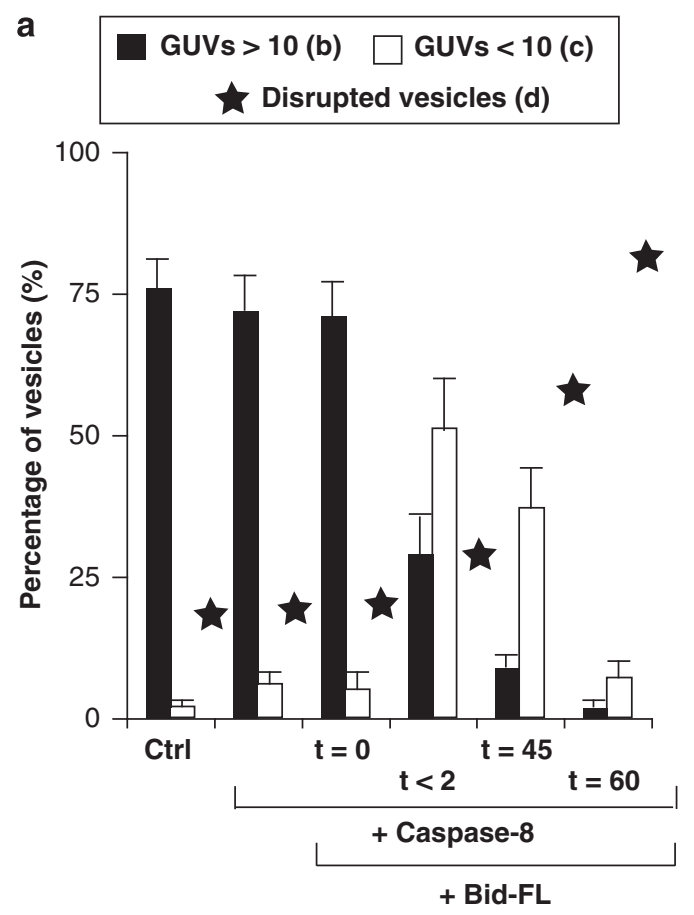

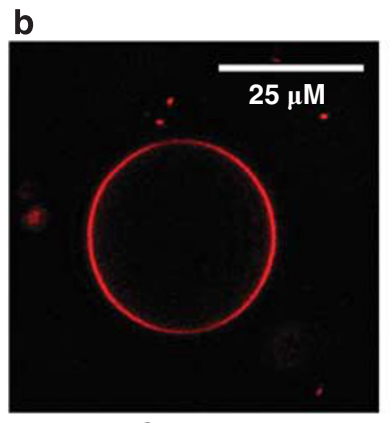

+ Caspase-8

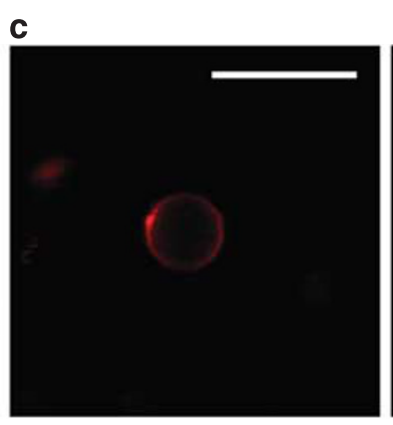

+ Caspase-8

+ Bid-FL

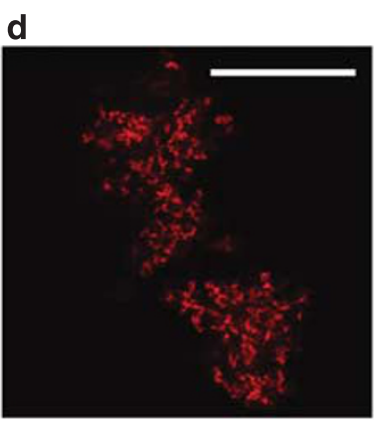

+ Caspase-8 + Bid-FL

Figure 5 CL-GUV distribution after formation of caspase-8/Bid-FL platform and image analysis. (a) The two types of GUVs (over $10 \mu \mathrm{m}$ and under $10 \mu \mathrm{m}$ ) and the fluorescent debris in various conditions and after various times (in minutes). In black, the high FSC GUVs and in white, the low FSC GUVs. (b-d) Confocal images after staining with DiD $(0.05 \%(\mathrm{~mol}))$. Caspase-8 $(500 \mathrm{nM})$ and FL-Bid-Alexa647 $(100 \mathrm{nM})$ were added sequentially to the GUV preparations. The debris results from the action of tBid produced by the newly formed enzymatic platform

apoptosis execution. ${ }^{34}$ In this work, we tested in a reconstituted system the following hypothesis: active caspase- 8 binds to a CL-enriched domain at mitochondrial contact sites in which it constitutes an activation platform, ${ }^{21}$ upon which active caspase-8 can recruit Bid-FL and process it to generate tBid.

First, we show that the combination of a new biometric system with a powerful quantitative technique - flow cytometry - allows systematic and statistically valid studies of active caspase-8 and Bid-FL interaction with lipid membranes. Using PC-GUVs containing or not containing $\mathrm{CL}$ as a model of mitochondrial contact sites, we were able to reproduce features of active caspase- 8 binding to the functionalized mitochondrial membranes recently described using Barth syndrome-derived cell lines. ${ }^{21}$ Investigations of the binding of active caspase- 8 is challenging in cells, but is facilitated in this experimental model. Caspase-8 preferentially binds to CL in GUVs and indeed its non-specific binding to PC-GUVs is weak. This is consistent with the view that active caspase- 8 is specifically targeted to mitochondria upon activation. This does not resolve the question of the affinity of the pro-active form of caspase-8 for CL. Nevertheless, such interaction appears to occur, followed by the recruitment of further pro-caspase-8 molecules that homodimerize and undergo the cleavage events necessary for caspase-8 activation. We showed that $C L$ strongly supports the binding of active caspase-8 to the GUVs. In this study, we have tested four concentrations of $\mathrm{CL}(5,10,15$ and $20 \%)$ and found that $5 \%$ of CL was sufficient to provide maximal binding of active caspase-8 to the GUVs (data not shown).

Our investigations are also informative concerning the possibility of constituting a complete platform composed of $\mathrm{CL} /$ caspase-8 and Bid-FL. After death receptor engagement, the activation of caspase-8 results in the cleavage of Bid-FL to give a truncated form of $\mathrm{tBid}^{35}{ }^{35} \mathrm{Bid}$ then translocates to 
mitochondria and induces the oligomerization of Bax/Bak; this leads to cytochrome $c$ release and activation of executioner caspase-3 and -9. Immunoelectron microcopy suggests that tBid is targeted in a CL-dependent manner to the contact sites. ${ }^{16,25}$ The minimal requirement of tBid binding to CL-enriched contact sites was shown to be $\alpha$-helices $6^{36}$ with a functional activity toward the respiratory chain linked to $\alpha$-helices $6-8 .{ }^{23}$ Note that tBid binds to $C L$ and $C L$ derivatives, such as monolysocardiolipin (MLCL). Consequently, even if the $C L$ is not mature, tBid can still bind MLCL that is sufficient for directing tBid translocation to the mitochondria.

We tested the effect of tBid binding to GUVs. We found unambiguously that tBid binds very efficiently and specifically to CL-GUVs at nM concentrations. Moreover, tBid binding and its subsequent effects were very fast and induced GUVs rupture followed by re-vesiculation or even total destruction. This is consistent with our recent results concerning the interaction between CL-containing lipid monolayers and the constitution of lipid microdomains induced by tBid (or by the hairpin-forming domain of tBid). ${ }^{23,24}$ As we can follow tBid generation on CL-GUVs, we now have a tool for testing the functional activity of a potential activation platform composed of CL/caspase-8/Bid-FL.

We found that Bid-FL alone was unable to bind to either PC-only-GUVs or CL-GUVs (even those containing $20 \% \mathrm{CL}$, result not shown). Although there is some controversy, we confirmed previous findings that Bid-FL does not bind to CL. ${ }^{33}$ Bid-FL bound to CL-GUVs only if active caspase- 8 was present at the vesicle surface. Active caspase- 8 binding to $C L$ is, in practice, non-saturable, this is also the case for Bid-FL. We found that the complexes formed very quickly and rapidly became enzymatically active; the process was too fast to be followed by imaging of GUVs by confocal microscopy. Within $2 \mathrm{~min}$, the CL-GUVs samples exhibited two subpopulations both stained by Bid-FL Alexa647, one similar to the initial or control GUVs and one composed of smaller GUVs with high SSC. After $45 \mathrm{~min}$ the initial CL-GUV population and its fluorescence associated with Bid-FL had been lost (because the truncated form is non-fluorescent). A third population of components was also generated by the activity of the platform: fluorescent debris from broken GUVs with high fluorescence and a tendency to aggregate.

Studies with our model vesicles indicate that active capase8 binds to $C L$, and Bid-FL subsequently binds to the fixed active caspase- 8 at its cleavage site leading to tBid generation. tBid is in turn the driving force destabilizing the vesicles, presumably through increased membrane curvature as described by Epand et al. ${ }^{26}$ Indeed, our approach, based on flow cytometry with the newly developed biomimetic model of mitochondrial contact sites, strongly supports the hypothesis of the formation of a platform composed of caspase- 8 and Bid-FL bound to CL. Our major finding is that Bid-FL requires the presence of caspase-8 for binding to GUVs, and this provides further insight into the nature of the so-called 'mitosome'-triggering complex.

\section{Materials and Methods}

Protein preparation. We prepared fluorescently labeled human tBID as described ${ }^{37}$ from BID complementary (CDNA) in pET15b encoding a single cysteine at position 64 in the tBID fragment, and labeled it with Alexa647 maleimide
(Invitrogen, Cergy-Pontoise, France). ${ }^{33}$ We purified full-length BID by the same protocol as that used for HBID, but from a CDNA with C15S and C28S mutations, and labeled it with Bodipy or Alexa647 maleimide. ${ }^{33} \mathrm{BCl}-\mathrm{X}_{\mathrm{L}} \Delta \mathrm{C}$ was prepared as described ${ }^{33}$ by Garcia-Saez et al. ${ }^{33}$

Preparation of GUVs. All lipids were from Avanti Polar Lipids (Alabaster, AL, USA). We prepared GUVs by the electroformation method. We spread $5 \mu \mathrm{l}$ of lipid mixture at $1 \mathrm{mg} / \mathrm{ml}$ in chloroform on two Pt wire electrodes. We immersed the lipid films in a chamber containing $300 \mathrm{mM}$ sucrose solution and connected the Pt wires to a power generator. Electroformation was at $2.3 \mathrm{~V}$ and $10 \mathrm{~Hz}$ for $1 \mathrm{~h}$ at room temperature, and the GUVs formed were released from the electrodes by changing the frequency to $2 \mathrm{~Hz}$ for $30 \mathrm{~min}^{33}$

Protein binding and image analysis. Aliquots of $50 \mu \mathrm{l}$ of the electroformed GUVs were sedimented in $500 \mu \mathrm{l}$ PBS containing the corresponding proteins. GUV labeling was assessed by confocal microscopy. We added $25 \mathrm{nM}$ of nonyl acridine orange (NAO) to reveal the presence or absence of $\mathrm{CL}$; after 30 min of incubation at room temperature the resulting fluorescence was measured in the green channel. DiD $\left(\mathrm{DilC}_{18}(5), 0.05 \%(\mathrm{~mol})\right)$, a lipophilic carbocyanine dye, was used to vizualize vesicles, imaged in the red channel, independently of their CL content. We used an LSM 510 Meta microscope (Zeiss, Jena, Germany) with a $40 \times 1.2$ NA C-Apochromat water objective (Zeiss) in the multi-track mode. UV/488/543/633 and $545 \mathrm{~nm}$ filters were used as main and secondary dichroics. The green channel consisted of an Ar laser with excitation wavelength at $488 \mathrm{~nm}$ and a 505-530-nm band-pass filter, whereas we configured the red channel with a 633-nm excitation laser and a 650-nm long-pass filter.

Flow cytometry analysis of GUVs. For flow cytometry analysis, preincubation when appropriate was performed with $10 \mu$ l of electroformed GUVs in $100 \mu \mathrm{l}$ of PBS (subsequently made up to $1 \mathrm{ml}$ in PBS for flow cytometry analysis). The pre-incubated GUVs were sedimented and the pellet was washed to eliminate non-specific binding of dyes and proteins. Some current experiments were performed on-line in the flow cytometer, in which case $10 \mu$ lof the GUV preparation was diluted to $1 \mathrm{ml}$ in PBS and then passed through the flow cytometer; the reaction was started by addition of proteins directly to the tube during data recording and the enzymatic activity was measured either as changes in light scatter or by fluorescence measurement. Forward low-angle light scatter (FSC) and side scatter (SSC) were recorded. We measured NAO fluorescence in the green channel. We checked that non-specific binding of proteins was limited in parallel experiments involving sedimentation of GUVs in dye- or protein-free media (data not shown).

We used CaspACE FITC-VAD-fmk (Promega, Charbonnieres, France), a fluorescent derivative of VAD-fmk, at $500 \mathrm{nM}$ to stain the active site of caspase-8 ( $100 \mathrm{nM}$, non-labeled) bound to GUVs. We performed control experiments with the non-fluorescent caspase-8 inhibitors Z-VAD- fmk $(5 \mu \mathrm{M})$, Boc-D-fmk (500 nM) and, more specific for caspase-8, Z-IETD-fmk (500 nM). The amount of FL-Bid Alexa647 added is always $100 \mathrm{nM}$.

We used a FACS Calibur4C (Becton-Dickinson, Franklin Lakes, NJ, USA) equipped with an argon laser at $488 \mathrm{~nm}$ and a red diode laser at $635 \mathrm{~nm}$. A $530 \pm 30 \mathrm{~nm}$ band pass filter was used for green fluorescence ( $448 \mathrm{~nm}$ excitation) and a $661 \pm 16 \mathrm{~nm}$ band pass for the red fluorescence (excitation $635 \mathrm{~nm}$ ). Beads of known size ( $10 \mu \mathrm{m}$ diameter) were added to the samples as size markers when appropriate. Given the strength of the light-scattering properties of the GUVs used in our study, we recorded the FSC and theSSC, and a particle threshold was set on the FSC to exclude debris and very small particles.

\section{Conflict of interest}

The authors declare no conflict of interest.

Acknowledgements. This work was supported by the Association Française contre les Myophaties (grant number 11557 and to PXP). We thank Jean-Claude Martinou (University of Geneva, Switzerland) for providing us with purified active caspase-8.

1. Nagata S. Fas ligand-induced apoptosis. Annu Rev Genet 1999; 33: 29-55.

2. Krammer PH. CD95's deadly mission in the immune system. Nature 2000; 407: 789-795. 
3. Peter ME, Budd RC, Desbarats J, Hedrick SM, Hueber AO, Newell MK et al. The CD95 receptor: apoptosis revisited. Cell 2007; 129: 447-450.

4. Strasser A, Jost PJ, Nagata $S$. The many roles of FAS receptor signaling in the immune system. Immunity 2009; 30: 180-192.

5. Wang K, Yin XM, Chao DT, Milliman CL, Korsmeyer SJ. BID: a novel BH3 domain-only death agonist. Genes Dev 1996; 10: 2859-2869.

6. Yin XM, Wang K, Gross A, Zhao Y, Zinkel S, Klocke B et al. Bid-deficient mice are resistant to Fas-induced hepatocellular apoptosis. Nature 1999; 400: 886-891.

7. McKenzie MD, Carrington EM, Kaufmann T, Strasser A, Huang DC, Kay TW et al. Proapoptotic $\mathrm{BH} 3$-only protein Bid is essential for death receptor-induced apoptosis of pancreatic beta-cells. Diabetes 2008; 57: 1284-1292.

8. Luo X, Budihardjo I, Zou H, Slaughter C, Wang X. Bid, a bcl-2 interacting protein, mediates cytochrome $c$ release from mitochondria in response to activation of cell surface receptors. Cell 1998; 94: 481-490.

9. Li H, Zhu H, Xu CJ, Yuan J. Cleavage of BID by caspase 8 mediates the mitochondrial damage in the Fas pathway of apoptosis. Cell 1998; 94: 491-501.

10. van Loo G, Saelens X, van Gurp M, MacFarlane M, Martin SJ, Vandenabeele P. The role of mitochondrial factors in apoptosis: a Russian roulette with more than one bullet. Cell Death Differ 2002; 9: 1031-1042.

11. Schlame M, Haldar D. Cardiolipin is synthesized on the matrix side of the inner membrane in rat liver mitochondria. J Biol Chem 1993; 268: 74-79.

12. Schlame M, Rua D, Greenberg ML. The biosynthesis and functional role of cardiolipin Prog Lipid Res 2000; 39: 257-288.

13. Ardail D, Privat JP, Egret-Charlier M, Levrat C, Lerme F, Louisot P. Mitochondrial contact sites. Lipid composition and dynamics. J Biol Chem 1990; 265: 18797-18802.

14. Schlame M. Cardiolipin synthesis for the assembly of bacterial and mitochondrial membranes. J Lipid Res 2008; 49: 1607-1620.

15. Gonzalvez F, Gottlieb E. Cardiolipin: setting the beat of apoptosis. Apoptosis 2007; 12 877-885.

16. Lutter M, Fang M, Luo X, Nishijima M, Xie X, Wang X. Cardiolipin provides specificity for targeting of tBid to mitochondria. Nat Cell Biol 2000; 2: 754-761.

17. Gonzalvez F, Pariselli F, Dupaigne P, Budihardjo I, Lutter M, Antonsson B et al. tBid interaction with cardiolipin primarily orchestrates mitochondrial dysfunctions and subsequently activates Bax and Bak. Cell Death Differ 2005; 12: 614-626.

18. Kagan E, Tyurin VA, Jiang JY, Tyurina YY, Ritov VB, Amoscato AA et al. Cytochromme $c$ acts as a cardiolipoin oxygenase required for release of proapoptotic factors. Nature Chemical Biology 2005; 1: 223-232.

19. Stegh AH, Herrmann H, Lampel S, Weisenberger D, Andra K, Seper M et al. Identification of the cytolinker plectin as a major early in vivo substrate for caspase 8 during CD95- and tumor necrosis factor receptor-mediated apoptosis. Mol Cell Biol 2000; 20: 5665-5679.

20. Stegh AH, Barnhart BC, Volkland J, Algeciras-Schimnich A, Ke N, Reed JC et al. Inactivation of caspase-8 on mitochondria of Bcl-xL-expressing MCF7-Fas cells: role for the bifunctional apoptosis regulator protein. J Biol Chem 2002; 277: 4351-4360.

21. Gonzalvez F, Schug ZT, Houtkooper RH, Mackenzie ED, Brooks DG, Wanders RJ et al. Cardiolipin provides an essential activating platform for caspase-8 on mitochondria. J Cell Biol 2008; 183: 681-696.

22. Gonzalvez F, Bessoule JJ, Rocchiccioli F, Manon S, Petit PX. Role of cardiolipin on tBid and tBid/Bax synergistic effects on yeast mitochondria. Cell Death Differ 2005; 12: 659-667.
23. Gonzalvez F, Pariselli F, Jalmar O, Dupaigne $P$, Sureau F, Dellinger $M$ et al. mechanistic issues of the interaction of the hairpin-forming domain of tBid with mitochondrial cardiolipin. PLoS One 2010; 5: v9342.

24. Petit PX, Dupaigne P, Gonzalvez F, Pariselli F, Bernard S. tBid-induced alterations of lipid monomalyers initially depends on $\mathrm{H} 6 / \mathrm{H} 7$ peptides forming hairpin domain-cardiolipin relationship: a biophysical approach. FEBS J 2009; 276: 6338-6354.

25. Lutter M, Perkins GA, Wang X. The pro-apoptotic Bcl-2 family member tBid localizes to mitochondrial contact sites. BMC Cell Biol 2001; 2: 22.

26. Epand RF, Martinou JC, Fornallaz-Mulhauser M, Hughes DW, Epand RM. The Apoptotic Protein tBid Promotes Leakage by Altering Membrane Curvature. J Biol Chem 2002; 277 : 32632-32639

27. Kim TH, Zhao Y, Ding WX, Shin JN, He X, Seo YW et al. Bid-cardiolipin interaction at mitochondrial contact site contributes to mitochondrial cristae reorganization and cytochrome c release. Mol Biol Cell 2004; 15: 3061-3072.

28. Liu J, Weiss A, Durrant D, Chi NW, Lee RM. The cardiolipin-binding domain of Bid affects mitochondrial respiration and enhances cytochrome $c$ release. Apoptosis 2004; 9 : 533-541.

29. Kischkel FC, Hellbardt S, Behrmann I, Germer M, Pawlita M, Krammer PH et al. Cytotoxicity-dependent Apo-1 (FAs/CD95)-associated proteins form a death-inducing signaling complex (DISC) with the receptor. EMBO J 1995; 14: 5579-5588.

30. Zou H, li Y, Liu X, Wang X. An APAF-1.cytochrome c multimeric complex is a functional apoptososome that activates procaspase-9. J Biol Chem 1999; 274: 11549-11556.

31. Martinon F, Burns K, Tschopp J. The inflammasome: a molecular platform triggering activation of inflammatory caspases and processing of prolL-beta. Mol Cell 2002; 10 : $417-426$.

32. Tinel A, Tschopp J. The PIDDosome, a protein complex implicated in activation of caspase2 in response to genotoxic stress. Science 2004; 304: 843-846.

33. Garcia-Saez AJ, Ries J, Orzaez M, Perez-Paya E, Schwille P. Membrane promotes tBID interaction with $\mathrm{BCL}(\mathrm{XL})$. Nat Struct Mol Biol 2009; 16: 1178-1185.

34. Scaffidi C, Fulda S, Srinivasan A, Friesen C, Li F, Tomaselli KJ et al. Two CD95 (APO-1/Fas) signaling pathways. Embo J 1998; 17: 1675-1687.

35. Gross A, Yin XM, Wang K, Wei MC, Jockel J, Milliman C et al. Caspase cleaved BID targets mitochondria and is required for cytochrome $c$ release, while $B C L-X L$ prevents this release but not tumor necrosis factor-R1/Fas death. J Biol Chem 1999; 274: 1156-1163.

36. Hu X, Han Z, Wyche JH, Hendrickson EA. Helix 6 of tBid is necessary but not sufficient for mitochondrial binding activity. Apoptosis 2003; 8: 277-289.

37. Zha J, Weiler S, Oh KJ, Wei MC, Korsmeyer SJ. Posttranslational N-myristoylation of $\mathrm{BID}$ as a molecular switch for targeting mitochondria and apoptosis. Science 2000; 290: 1761-1765.

Cell Death and Disease is an open-access journal published by Nature Publishing Group. This work is licensed under the Creative Commons Attribution-Noncommercial-Share Alike 3.0 Unported License. To view a copy of this license, visit http:// creativecommons.org/licenses/by-nc-sa/3.0/ 